\title{
Selecting persistent atrial fibrillation patients for pulmonary vein isolation based on the response to amiodarone: efficacy of the "one step back" strategy
}

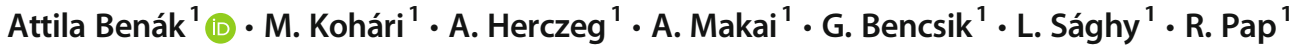

Received: 5 August 2018 / Accepted: 28 January 2019

(C) Springer Science+Business Media, LLC, part of Springer Nature 2019

\begin{abstract}
Purpose Pulmonary vein isolation (PVI) by catheter ablation has reduced efficacy for the treatment of persistent atrial fibrillation (persAF), as compared to paroxysmal atrial fibrillation (paroxAF). We investigated whether the selection of persAF patients for PVI who "step back" to the paroxysmal stage on amiodarone offers a success rate comparable to that of patients with paroxAF. Methods Sixty-two consecutive persAF patients and 62 matched control patients with paroxAF were included. Persistent patients were started on amiodarone and cardioverted to sinus rhythm (SR). PVI was performed after 3 months in those who "stepped back" and had sustained SR and in all paroxAF patients.

Results Five of the $62(8 \%)$ study patients returned to persAF after cardioversion; despite amiodarone, they did not undergo PVI. The rest received PVI and was followed for a mean of $31 \pm 14$ months. Redo procedures were performed in $44 \%$ and $29 \%$ in the persAF and paroxAF group $(p=0.093)$, respectively. The recurrence rate after multiple procedures without antiarrhythmic drugs was similar among the persAF and paroxAF patients $(11 \%$ and $7 \%)$ at 6 months $(p=0.510)$, but increased in the persAF group at 1 year $(21 \%$ and $9 \%, p=0.065)$ and exceeded that of the paroxAF group at the end of the follow-up (26\% and $12 \%, p=0.046)$. Kaplan-Meier survival analysis showed shorter time to recurrence in the persAF group $(p=0.045)$.

Conclusion PersAF patients who "step back" to the paroxysmal stage on amiodarone can expect long-term success of a PVI-only strategy in more than $70 \%$ of the time. However, late recurrences are more common compared to paroxAF.
\end{abstract}

Keywords Pulmonary vein isolation $\cdot$ Ablation $\cdot$ Amiodarone $\cdot$ Persistent atrial fibrillation $\cdot$ Atrial reverse remodeling

\section{Introduction}

Pulmonary vein isolation (PVI) by catheter ablation has reduced efficacy for the treatment of persistent atrial fibrillation (persAF), as compared to paroxysmal atrial fibrillation (paroxAF) [1]. In the mechanism of the latter, triggers from the pulmonary veins (PVs) have a dominant role [2]. However, there is a considerable progression of atrial fibrillation (AF) over time, characterized by remodeling of the atria and persistence of AF by extrapulmonary sources $[3,4]$. Several techniques have been developed to address

Attila Benák

benakattila@gmail.com

1 Second Department of Internal Medicine and Cardiology Center, University of Szeged, 8. Semmelweis Street, Szeged 6725, Hungary extrapulmonary sources, including left atrial (LA) linear ablation and electrogram-based ablation; however, these have not consistently improved outcomes when added to PVI $[1,5]$. Previous studies have suggested that a better outcome of PVI may be expected in those persAF patients who can be maintained in sinus rhythm (SR) by aggressive antiarrhythmic drug (AAD) pretreatment, compared with those who return to persAF despite such efforts $[6,7]$. Therefore, another approach to improve ablation success in persAF would be the selection of patients for PVI, whose atrial remodeling is reversible and is able to "step back" to the paroxysmal stage on AAD. Amiodarone has superior efficacy in AF and has been shown to be able to reverse atrial remodeling in an animal model $[8,9]$. Therefore, we tested the hypothesis that when pretreatment of persAF patients by amiodarone results in a "step back" to the paroxysmal stage, the efficacy of PVI would be comparable to that of patients with true paroxAF. 


\section{Methods}

\subsection{Patients}

The study group consisted of consecutive patients with symptomatic, persistent atrial fibrillation (persAF) resistant to AAD therapy, other than amiodarone. No study patient has been on amiodarone previously. PersAF was defined as an arrhythmia lasting more than 1 week according to the European Society of Cardiology [10]. Patients with prior LA ablation, reduced left ventricular ejection fraction, and valvular heart disease and aversion or a contraindication for amiodarone were excluded. No patient with documented persAF lasting more than 1 year was included.

For the control group, we selected consecutive patients with symptomatic paroxAF, from a contemporaneous population undergoing their first pulmonary vein isolation (PVI), after the failure of rhythm control with propafenone or sotalol. Controls were matched with the study group in age, gender, comorbidities, and echocardiographic LA dimension to ensure the same distribution of these characteristics in the two groups. There was no patient previously on amiodarone in the control group.

\subsection{Periprocedural antiarrhythmic drug protocol}

Amiodarone was initiated for the patients with persAF after transoesophageal echocardiography (TOE) ruled out intracardiac thrombi. The drug was administered in a dose of $800 \mathrm{mg} /$ day during the first week, $400 \mathrm{mg} /$ day during the second week, and $200 \mathrm{mg}$ afterwards. After at least 1 month of amiodarone treatment, patients underwent electrical cardioversion, if still in persAF. Patients without recurrence of persAF during the ensuing 2 months underwent PVI. Amiodarone was continued for the 8-week blanking period after PVI and stopped thereafter. In the control group, any AAD therapy was discontinued before the procedure and reinitiated only if recurrences occurred. Therefore, no patient without recurrences continued to receive AADs beyond the blanking period.

\subsection{Pulmonary vein isolation}

After providing written informed consent according to institutional protocols, all patients underwent antral pulmonary vein isolation in sinus rhythm (SR), without any additional linear ablation, except a right atrial flutter line in patients with documented or induced typical atrial flutter. Radiofrequency energy was applied with a deflectable irrigated catheter, using a point-by-point technique. The procedures were guided by a real-time 3D non-fluoroscopic navigation system (NavX or CARTO) and intracardiac echocardiography. A circular mapping catheter was used for the recording of PV potentials and the verification of the isolation. Complete isolation of all the
PVs after a 30-min waiting period was the endpoint of the procedure.

\subsection{Patient follow-up}

Patients had follow-up visits 3 months, 6 months, and 1 year after the procedure, and yearly thereafter. Symptom status, 12lead ECG, 24-h or 1-week Holter ECG, and transtelephonic ECG were used for assessing AF recurrence. All patients without symptoms underwent 1-week Holter monitoring $15 \pm$ 2 months (median 15 months) after the last procedure to test for asymptomatic recurrences. Recurrences were defined as any atrial arrhythmia lasting more than $30 \mathrm{~s}$. Success was defined as no recurrences beyond the blanking period off all AADs. Patients who had their AADs reinitiated because of recurrences were considered to have failed PVI. In case of documented AF recurrence, a redo procedure was recommended for symptomatic patients. During redo PVI, all reconnected PVs were reisolated, and it was followed by the same outpatient care protocol as the index procedure. In case of redo ablation, the follow-up time was calculated after the last procedure. Patients who had not completed the 6-month follow-up after the redo PVI were excluded from the multipleprocedure analysis and were considered failures in the singleprocedure analysis.

\subsection{Statistical analysis}

IBM SPSS Statistics 23 was used for statistical analysis. Continuous variables are indicated as mean $\pm \mathrm{SD}$, and categorical variables are expressed in percentage form. The difference between the study and the control group was tested with Student's $t$ test in case of continuous variables and the chisquare test was used for the categorical variables. We used the Cox regression to estimate hazard ratios (HR) and 95\% confidence intervals (CI) for arrhythmia recurrence in the two groups at 6 months, 1 year, and at the end of the follow-up. The time to recurrence during the follow-up period was tested by the Kaplan-Meier curve with the log-rank analysis. Logistic regression was utilized to determine the predictor factors of AF recurrence. $p<0.05$ was considered statistically significant.

\section{Results}

\subsection{Patient characteristics}

Sixty-two consecutive patients were included in the study group. They all had ongoing persAF at the time of inclusion (mean duration, $3.8 \pm 3.9$ months; median, 2 months). Twenty of them $(32 \%)$ had previously undergone cardioversion for persAF; for the rest, the qualifying persistent episode was 
the first one. None of them was experiencing concurrent paroxAF episodes. All of the included patients had successful cardioversion after amiodarone loading; however, 5 patients $(8 \%)$ returned to persAF during the subsequent 2 months and did not undergo ablation. The remaining 57 patients "stepped back" from persAF to having only paroxysmal episodes or sustained SR with amiodarone treatment and underwent PVI as per protocol.

There was no difference between the study patients and the control group $(N=62)$ in demographic data, comorbidities, and echocardiographic parameters. Left ventricular ejection fraction, antero-posterior left atrial diameter, grade of spontaneous left atrial echo contrast, and the flow velocity of the left atrial appendage on TOE were investigated. All persAF patients were studied during ongoing arrhythmia (before cardioversion), while paroxAF patients were generally studied in SR (Table 1).

The mean follow-up time was $30.2 \pm 13.6$ vs. $29.8 \pm$ 14.4 months after the first procedure and $23.13 \pm 13.4$ vs. $24.9 \pm 13.9$ months after the last procedure, in the study and control groups, respectively ( $p=\mathrm{NS}$ ). Two patients were lost after 6 months to follow-up; otherwise, all patients completed at least 1-year follow-up.

The rate of redo procedures tended to be higher in the study group (44\% vs. $29 \%, p=0.093$ ).

\subsection{Efficacy of pulmonary vein isolation}

Isolation of all four pulmonary veins (PVI) was achieved in all patients at the index procedure in both groups. After the first procedure, $56 \%$ of patients achieved complete arrhythmia freedom without AAD. In the control group, the single procedure success was significantly better compared with the persistent group $(64.5 \%$ vs. $44 \%, p=0.028)$. In five patients (4\%), rhythm control strategy was abandoned after the first procedure due to the lack of symptoms, patients' preferences, or severe comorbidities. Redo procedures were performed in $44 \%(n=25)$ of persAF and $29 \%(n=18)$ of paroxAF patients
( $p=0.093)$, respectively. PV reconnection was observed in $95.8 \%$ in persAF and $93.3 \%$ in paroxAF patients. The number of reconnected PVs was comparable in the two groups $(2.2 \pm$ 1.1 vs. $1.9 \pm 1, p=0.463)$. A second redo PVI was done in three cases, all of which showed extensive PV reconnections during the first redo PVI. The total number of ablation procedures was 84 in persAF group and 81 in paroxAF group. After the last procedure, at 6-month follow-up, there was no statistically significant difference in the recurrence rate between the two groups $(11 \%$ vs. $7 \%, p=0.510 ; \mathrm{HR}, 1.87$; 95\%CI, 0.528 6.639). The difference started to emerge at the 12-month follow-up (21\% vs. $9 \%, p=0.065$; HR, 2.648; 95\%CI, $0.905-$ 7.750) and became significant by the end of the follow-up (26\% vs. $12 \%, p=0.046$; HR, 2.52; 95\%CI, 1.003-6.324). The Kaplan-Meier survival curves after the first and multiple procedures are shown in Figs. 1 and 2. ParoxAF patients showed significantly longer mean time to recurrence compared with persAF patients $(26.6 \pm 1.8$ vs. $22.7 \pm 1.7$ months after the first procedure and $33.2 \pm 1.2$ vs. $29.4 \pm 1.8$ months after the last procedure, respectively, $p=0.045$ ).

In a multivariable analysis including demographic data, comorbidities, and echocardiographic parameters, only age emerged as a significant predictor for the failure of PVI (Table 2).

\section{Discussion}

Pulmonary vein (PV) isolation (PVI) is currently the only validated approach for the non-pharmacologic treatment of persistent atrial fibrillation (persAF). However, the success of PVI in persAF remains limited when compared with paroxysmal atrial fibrillation (paroxAF). The lower success rate in persAF is likely due to adverse remodeling of the atria and emergence of non-PV sources of AF. There is evidence however that atrial remodeling can be reversed when sinus rhythm (SR) is restored for prolonged periods [11]. Previous studies have suggested a better outcome of PVI in persAF patients,
Table 1 Baseline clinical characteristics and echocardiographic parameters

\begin{tabular}{llll}
\hline & Persistent AF $(N=57)$ & Paroxysmal AF $(N=62)$ & $p$ value \\
\hline Male gender (\%) & 68.4 & 69.4 & 0.611 \\
Hypertension (\%) & 89.5 & 80.6 & 0.208 \\
Age (years) & $62.7 \pm 8.6$ & $61.21 \pm 7.7$ & 0.315 \\
Diabetes mellitus (\%) & 19 & 16.1 & 0.683 \\
Coronary disease (\%) & 5.2 & 6.5 & 0.765 \\
Ejection fraction (\%) & $60.8 \pm 8.7$ & $62.5 \pm 6.3$ & 0.242 \\
LA diameter (mm) & $48.25 \pm 7.17$ & $47.38 \pm 4.24$ & 0.384 \\
LA appendage flow (cm/s) & $23.36 \pm 8.05$ & $25.27 \pm 15.9$ & 0.549 \\
SEC in LA appendage (\%) & 19.6 & 10.7 & 0.517 \\
AF history (months) & $46.6 \pm 47.8$ & $61.6 \pm 76$ & 0.223 \\
\hline
\end{tabular}

$A F$, atrial fibrillation; $L A$, left atrium; $S E C$, spontaneous echo contrast 
Fig. 1 Kaplan-Meier survival curve of AF recurrence after a single procedure and hazard ratios for arrhythmia recurrence at 6 , 12 months, and at the end of the follow-up

Fig. 2 Kaplan-Meier survival curve of AF recurrence after multiple procedures and hazard ratios for arrhythmia recurrence at 6,12 months, and at the end of the follow-up

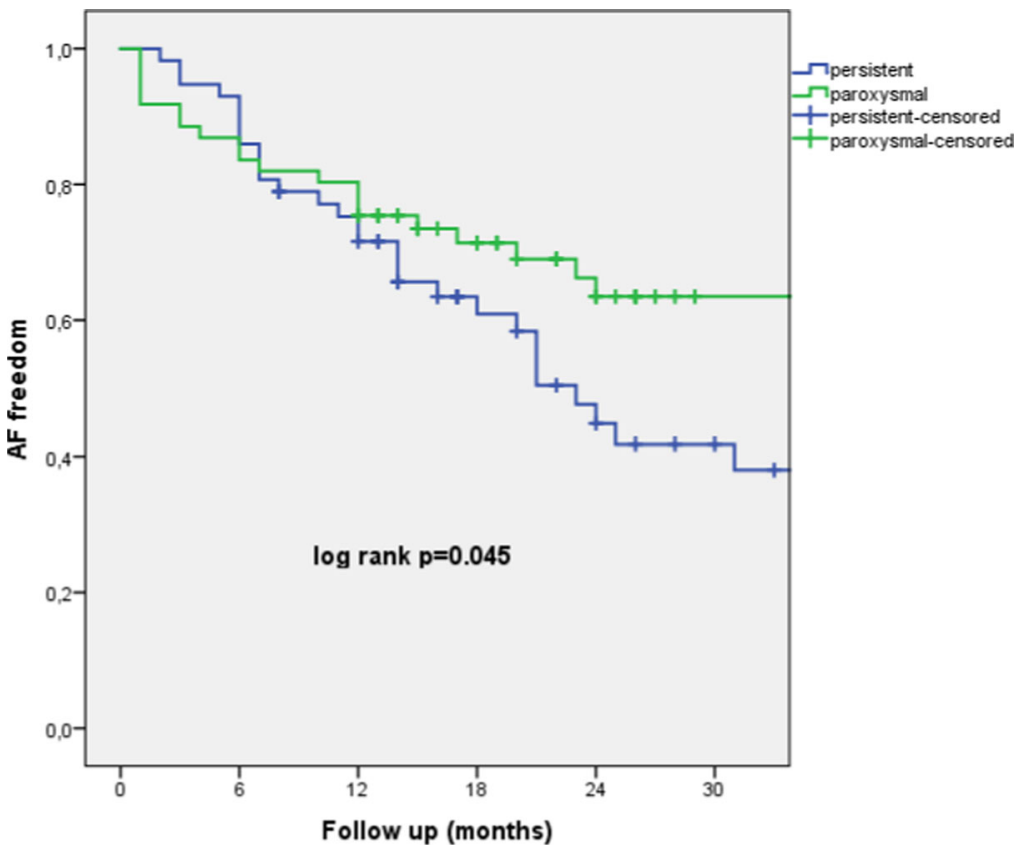

\begin{tabular}{lcccccc}
\multicolumn{9}{c}{ Patients at risk } & & & & \\
paroxysmal & 62 & 51 & 44 & 32 & 21 & 14 \\
persistent & 57 & 49 & 38 & 24 & 15 & 11 \\
& & & & & & \\
HR (p value) & & $1.312(0.592)$ & $1.145(0.718)$ & $1.58(0.105)$ \\
CI 95\% & & $0.486-3.539$ & $0.549-2.386$ & $0.908-2.778$
\end{tabular}

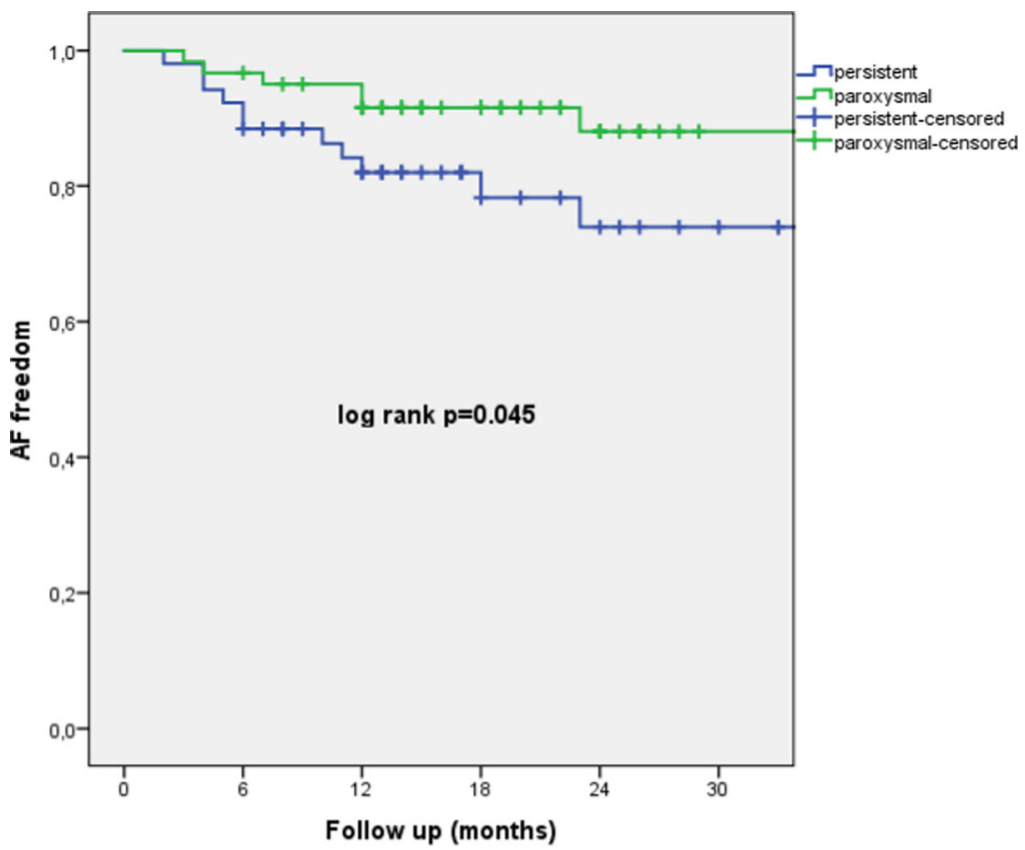

\begin{tabular}{lcccccc}
\multicolumn{9}{c}{ Patients at risk } & & & & \\
paroxysmal & 62 & 59 & 49 & 35 & 22 & 15 \\
\multicolumn{1}{c}{ persistent } & 52 & 46 & 35 & 21 & 17 & 13 \\
& & & & & & \\
HR (p value) & & $1.87(0.332)$ & $2.648(0.076)$ & $2.52(0.049)$ \\
CI 95\% & & $0.528-6.639$ & $0.905-7.750$ & $1.003-6.324$
\end{tabular}


Table 2 Clinical and echocardiographic predictors of AF recurrence

\begin{tabular}{|c|c|c|c|c|}
\hline & \multicolumn{2}{|l|}{ Univariate analysis } & \multicolumn{2}{|l|}{ Multivariate analysis } \\
\hline & OR $(95 \% \mathrm{CI})$ & $p$ value & OR $(95 \% \mathrm{CI})$ & $p$ value \\
\hline Male gender & $2.68(0.996-7.210)$ & 0.051 & $1.99(0.707-5.645)$ & 0.191 \\
\hline Age (years) & $1.09(1.018-1.168)$ & 0.013 & $1.075(1.002-1.154)$ & 0.043 \\
\hline Hypertension & $0.59(0.121-2.755)$ & 0.578 & & \\
\hline Diabetes mellitus & $4.56(0.572-36.342)$ & 0.152 & & \\
\hline Coronary disease & $1.31(0.149-11.527)$ & 0.808 & & \\
\hline AF type (persistent) & $2.57(0.939-7.038)$ & 0.066 & $2.25(0.790-6.405)$ & 0.129 \\
\hline LA diameter & $1.01(0.969-1.108)$ & 0.300 & & \\
\hline Ejection fraction & $1.026(0.958-1.098)$ & 0.467 & & \\
\hline LA appendage flow & $0.968(0.905-1.035)$ & 0.344 & & \\
\hline SEC in LA appendage & $2.4(0.604-9.541)$ & 0.214 & & \\
\hline AF history & $1.00(0.993-1.008)$ & 0.941 & & \\
\hline Redo procedure & $1.72(0.643-4.589)$ & 0.281 & & \\
\hline
\end{tabular}

$A F$, atrial fibrillation; $L A$, left atrium; $S E C$, spontaneous echo contrast when pretreatment by antiarrhythmic drugs (AADs) resulted in restoration of SR [6, 7]. Amiodarone has superior efficacy in $\mathrm{AF}$ and reverses electrical, as well as structural remodeling in an animal model [9], an effect not seen with class I and III AADs [8]. Based on these data, we selected persAF patients for PVI by the ability of amiodarone to result in a "step back" to the paroxysmal stage and compared the outcome of PVI with a matched cohort of paroxAF. In this population of lasting persAF, most patients "stepped back" on amiodarone. They have undergone a mean of 1.46 procedures to permanently isolate the PVs. No other ablation lesions were delivered, except for a CTI line in case of typical atrial flutter documented before or during the procedure. Compared with a matched paroxAF population undergoing the same procedure, the persAF patients had comparable short-term freedom from $\mathrm{AF}$, but more late recurrences. This underlines that atrial remodeling in persAF may be partly irreversible and patients with a history of persAF, even if temporarily "stepped back" by AADs, are in a higher risk category for late recurrences after PVI.

\subsection{Comparison with previous studies}

Two previous studies had indicated improved efficacy of PVI in persAF when pretreatment with AADs results in predominant SR. The first was a retrospective study of patients treated with the class III AAD bepridil before PVI [6]. Those who converted to SR on bepridil had higher AF-free rate (87\%) without AADs after PVI, compared with those who did not $(29 \%)$. In the second study, bepridil or amiodarone was combined with class I AADs (flecainide, aprindine, pilsicainide, or propafenone) before PVI [7]. Similarly, restoration of SR by AAD treatment predicted a more favorable outcome ( $61 \%$ vs. $22 \%)$. These results have raised hope that pretreatment of persAF patients by AADs to restore SR may achieve a similarly high success rate for PVI as in the case of paroxAF.

Khan et al. converted 71 persAF patients to SR and started on dofetilide 3 months prior to ablation [12]. Only two of them returned to persAF; they did not respond to PVI. The success rate in the remaining was not statistically different from the control group of paroxAF patients at 12 months post-PVI (70\% vs. $75 \%$ ). Our results contrast with those of Khan et al., in that paroxAF patients had a more favorable longterm outcome after PVI compared with persAF, even when AAD pretreatment resulted in predominant SR. In our series, paroxAF patients had considerably higher success at 6- and 12-months post procedure compared with those in Khan's study if we take into account our multiple-procedure success rate. Since only PVI was done during the first and all subsequent procedures, a redo PVI was considered to be part of the overall ablation effort and not a failure in our multipleprocedure analysis. A more than double redo rate in our study, compared with Khan's, also reflects our commitment to achieve permanent PVI in all patients. These differences along with improvements in technology may explain the higher success rate that is more in line with contemporary series of paroxAF [13-15]. Further explanation for the contrasting results may lie in our patients having more dilated LA and longer follow-up, which may enhance the difference between groups. We followed our patients beyond 12 months because the remarkably long half-life of amiodarone could obscure the difference in a shorter timeframe.

Interestingly, none of abovementioned studies have utilized amiodarone for reversing atrial remodeling before PVI in persAF, despite its proven anti-remodeling action in animal studies $[8,9]$. 


\subsection{The success of $\mathrm{PVI}$ alone in persAF}

There is an ongoing debate on the role of additional ablation beyond PVI. Despite incremental benefit shown in nonrandomized studies implementing linear and electrogram-based ablation, recent randomized trials suggested no role for these approaches [5, 16-18]. Therefore, PVI remains the only proven strategy both for paroxAF and persAF. However, up to half of the unselected patients experience a recurrence of persAF after PVI, compared with the $80-90 \%$ success rate in paroxAF $[1,13-15]$. Recent randomized trials, using modern technology for PVI in persAF, report success rates beyond 1 year, after multiple procedures, without AADs to be $40-60 \%$ [5, 18-21]. Our result in persAF patients selected by achieving "one step back" to a paroxysmal stage on amiodarone compares favorably with these and approaches the 12-month success of PVI in paroxAF.

\subsection{Limitations}

The main limitation of this study is that persAF patients not responding to amiodarone did not undergo ablation; therefore, one cannot be sure that a "step back" on amiodarone guarantees a higher success rate of PVI, compared with those who cannot be "stepped back." However, this concept is clearly supported by the abovementioned studies of AAD pretreatment before PVI. In these, the success rate was between 0 [12] and 22-29\% [6, 7] in patients who returned to persAF despite AAD treatment. Moreover, performing PVI in SR has been shown to result in a more favorable outcome, as opposed to in persAF [21]. We felt it unethical to offer the procedure to those who did not "step back" considering such an unfavorable outcome. On the other hand, the success rates reported in the above studies among patients who "stepped back" on AAD (61-87\%) and in our study are higher than in most reports of an unselected persAF population undergoing PVIonly and approaches the success seen in paroxAF. The definite answer would however require a prospective, randomized comparison.

Our study is also limited by the fact that efforts to better characterize the atrial substrate and the changes it may show during the study were not done, and echocardiograms were only routinely available before initiation of amiodarone therapy in persAF patients. Therefore, the change in LA volume during sustained SR that might reflect reverse remodeling could not be documented. Further limitation may be that persAF patients were on amiodarone throughout the procedure, while paroxAF patients were without AAD therapy. However, both groups underwent the same, anatomically determined PVI, without any effort to induce AF triggers. Therefore, the presence of AAD during the procedure would not be expected to have any influence on the long-term efficacy of PVI. Another limitation may be that since continuous rhythm monitoring by an implantable monitor after the procedure was not performed, recurrent, asymptomatic episodes of paroxAF may be less likely to be detected than persAF, possibly leading to a higher perceived success in the control group. However, prolonged ECG monitoring for 7 days was the standard for patients without symptoms to uncover asymptomatic recurrences.

\section{Conclusions}

Selecting persAF patients for PVI based on the favorable response to pretreatment with amiodarone-resulting in a "step back" to the paroxysmal stage - results in a long-term success rate above $70 \%$, which is higher than reported in most previous studies of PVI-only, offering a good strategy to improve ablation success in persAF. This strategy might have an even greater benefit in patients with more advanced disease than those in our study (e.g., long-standing persAF), whose "step back" rate is likely lower. However, late recurrences are more common in "stepped back," compared with true paroxAF patients, underlining the more advanced nature of their disease. This is important when consulting patients with a history of persAF, even if predominantly in SR on AADs, about the expected efficacy of PVI. Furthermore, after the procedure, more vigilance is required in following these patients.

\section{Compliance with ethical standards}

Conflict of interest The authors declare that they have no conflict of interest.

Ethical approval All procedures performed in studies involving human participants were in accordance with the ethical standards of the institutional research committee and with the 1964 Helsinki declaration and its later amendments or comparable ethical standards.

Informed consent Informed consent was provided according to institutional protocols.

Publisher's note Springer Nature remains neutral with regard to jurisdictional claims in published maps and institutional affiliations.

\section{References}

1. Parkash R, Tang AS, Sapp JL, Wells G. Approach to the catheter ablation technique of paroxysmal and persistent atrial fibrillation: a meta-analysis of the randomized controlled trials. J Cardiovasc Electrophysiol. 2011;22(7):729-38.

2. Haïssaguerre M, Jaïs P, Shah DC, Takahashi A, Hocini M, Quiniou $\mathrm{G}$, et al. Spontaneous initiation of atrial fibrillation by ectopic beats originating in the pulmonary veins. N Engl J Med. 1998;339(10): 659-66.

3. Nattel S, Burstein B, Dobrev D. Atrial remodeling and atrial fibrillation: mechanisms and implications. Circ Arrhythm Electrophysiol. 2008;1(1):62-73. 
4. Padfield GJ, Steinberg C, Swampillai J, Qian H, Connolly SJ, Dorian P, et al. Progression of paroxysmal to persistent atrial fibrillation: 10-year follow-up in the Canadian Registry of Atrial Fibrillation. Heart Rhythm. 2017 Jun;14(6):801-7.

5. Verma A, Jiang CY, Betts TR, Chen J, Deisenhofer I, Mantovan R, et al. Approaches to catheter ablation for persistent atrial fibrillation. N Engl J Med. 2015;372(19):1812-22.

6. Miyazaki S, Kuwahara T, Kobori A, Takahashi Y, Takei A, Sato A, et al. Pharmacological cardioversion preceding left atrial ablation: bepridil predicts the clinical outcome following ablation in patients with persistent atrial fibrillation. Europace. 2009;11:1620-3.

7. Igarashi M, Tada H, Sekiguchi Y, Yamasaki H, Arimoto T, Kuroki $\mathrm{K}$, et al. Effect of restoration of sinus rhythm by extensive antiarrhythmic drugs in predicting results of catheter ablation of persistent atrial fibrillation. Am J Cardiol. 2010;106(1):62-8.

8. Shinagawa K, Shiroshita-Takeshita A, Schram G, Nattel S. Effects of antiarrhythmic drugs on fibrillation in the remodeled atrium: insights into the mechanism of the superior efficacy of amiodarone. Circulation. 2003;107(10):1440-6.

9. Ashikaga K, Kobayashi T, Kimura M, Owada S, Sasaki S, Iwasa A, et al. Effects of amiodarone on electrical and structural remodeling induced in a canine rapid pacing-induced persistent atrial fibrillation model. Eur J Pharmacol. 2006;536(1-2):148-53.

10. Kirchhof P, Benussi S, Kotecha D, Ahlsson A, Atar D, Casadei B, et al. 2016 ESC Guidelines for the management of atrial fibrillation developed in collaboration with EACTS. Eur Heart J. 2016;37(38): 2893-962.

11. Slotwiner D, Steinberg J. Limited ablation for persistent atrial fibrillation using preprocedure reverse remodelling. Arrhythm Electrophysiol Rev. 2014;3(2):101-6

12. Khan A, Mittal S, Kamath GS, Garikipati NV, Marrero D, Steinberg JS. Pulmonary vein isolation alone in patients with persistent atrial fibrillation: an ablation strategy facilitated by antiarrhythmic drug induced reverse remodeling. J Cardiovasc Electrophysiol. 2011;22(2):142-8.

13. Gökoğlan Y, Mohanty S, Güneș MF, Trivedi C, Santangeli P, Gianni C, et al. Pulmonary vein antrum isolation in patients with paroxysmal atrial fibrillation: more than a decade of follow-up. Circ Arrhythm Electrophysiol. 2016;9(5):e003660.
14. Andrade JG, Monir G, Pollak SJ, Khairy P, Dubuc M, Roy D, et al. Pulmonary vein isolation using contact force ablation: the effect on dormant conduction and long-term freedom from recurrent atrial fibrillation - a prospective study. Heart Rhythm. 2014;11:1919-24.

15. Marijon E, Fazaa S, Narayanan K, et al. Real-time contact force sensing for pulmonary vein isolation in the setting of paroxysmal atrial fibrillation: procedural and 1-year results. J Cardiovasc Electrophysiol. 2014;25:130-7.

16. Zhang $\mathrm{Z}$, Letsas $\mathrm{KP}$, Zhang $\mathrm{N}$, Efremidis $\mathrm{M}, \mathrm{Xu} \mathrm{G}, \mathrm{Li} \mathrm{G}$, et al. Linear ablation following pulmonary vein isolation in patients with atrial fibrillation: a meta-analysis. Pacing Clin Electrophysiol. 2016;39(6):623-30.

17. Scott PA, Silberbauer J, Murgatroyd FD. The impact of adjunctive complex fractionated atrial electrogram ablation and linear lesions on outcomes in persistent atrial fibrillation: a meta-analysis. Europace. 2016;18(3):359-67.

18. Fink T, Schlüter M, Heeger CH, Lemes C, Maurer T, Reissmann B, Riedl J, Rottner L, Santoro F, Schmidt B, Wohlmuth P, Mathew S, Sohns C, Ouyang F, Metzner A, Kuck KH. Stand-alone pulmonary vein isolation versus pulmonary vein isolation with additional substrate modification as index ablation procedures in patients with persistent and long-standing persistent atrial fibrillation: the randomized Alster-lost-AF trial (ablation at St. Georg Hospital for Long-Standing Persistent Atrial Fibrillation). Circ Arrhythm Electrophysiol 2017;10(7). https://doi.org/10.1161/CIRCEP.117. 005114.

19. Dixit S, Marchlinski FE, Lin D, Callans DJ, Bala R, Riley MP, et al. Randomized ablation strategies for the treatment of persistent atrial fibrillation: RASTA study. Circ Arrhythm Electrophysiol. 2012;5(2):287-94.

20. Vogler J, Willems S, Sultan A, Schreiber D, Lüker J, Servatius H, et al. J Am Coll Cardiol. 2015;66(24):2743-52.

21. Bassiouny M, Saliba W, Hussein A, Rickard J, Diab M, Aman W, et al. Randomized study of persistent atrial fibrillation ablation: ablate in sinus rhythm versus ablate complex-fractionated atrial electrograms in atrial fibrillation. Circ Arrhythm Electrophysiol. 2016;9(2):e003596. 